\title{
Quantum Efficiency of Quantum Dot Lasers
}

\author{
Peter Blood, Fellow, IEEE
}

\begin{abstract}
The quantum efficiency relates the calculated to the measured external threshold current of a laser. This quantity is often estimated from the length dependence of the external differential efficiency above threshold, assuming the carrier density is pinned. Often it is also assumed that the internal current varies linearly with the external current; it is shown here this is not the case due to the effect of stimulated emission on the current-voltage relation of the active region. Furthermore, it has been observed that spontaneous emission from inhomogeneous quantum dots does not pin above threshold which questions the determination of their optical loss. This nonpinning is reproduced by a model in which the laser photon rate equation is coupled to rate equations for the occupation of dot states mediated by a thermal phonon distribution. The threshold current from this model agrees with a Fermi-Dirac calculation but the external efficiency above threshold is lower and its length dependence gives a smaller value of mode loss than the input value. The reasons for this behavior are analyzed and it is concluded that a Fermi-Dirac calculation does not represent the light-current characteristics in quantum dot lasers at room temperature.
\end{abstract}

Index Terms-Quantum dots, semiconductor lasers, spontaneous emission.

\section{INTRODUCTION}

K NOWLEDGE of the quantum efficiency of a diode laser is necessary when making a comparison between calculated gain-current relations and experimentally measured properties particularly the threshold current. Calculations usually provide the current due to intrinsic, radiative recombination processes within the active region ${ }^{1}\left(J_{\text {calc }}\right)$ whereas measurements on laser structures give the current supplied by an external source $\left(J_{e x t}\right)$ which includes that due to carrier leakage and recombination in other parts of the structure in addition to the active region. The relation between them is expressed as an efficiency by the deceptively simple relation $J_{e x t}=J_{\text {calc }} / \eta$.

In general the relation between the external current supplied and the internal radiative recombination current is made up of two components:

1) the fraction of the external current which enters the active region termed the injection efficiency, the balance being lost by current spreading and carrier leakage, and

2) the fraction of the recombination current in the active region which is due to radiative recombination termed the internal radiative efficiency, the balance being lost by non-radiative processes.

Manuscript received January 30, 2017; revised March 14, 2017; accepted March 19, 2017. Date of publication March 24, 2017; date of current version April 17, 2017. (Corresponding author: Peter Blood.)

The author is with the School of Physics and Astronomy, Cardiff University, Cardiff CF24 3AA U.K. (e-mail: bloodp@cf.ac.uk).

Digital Object Identifier 10.1109/JSTQE.2017.2687039

${ }^{1}$ Although Auger recombination is also an intrinsic process it is not usually included in the calculation.
It has been common practice to obtain the injection efficiency from an analysis of the differential quantum efficiency above threshold for devices of different cavity length, a procedure which also provides a value for the internal optical mode loss, $\alpha_{i}$. Setting aside uncertainty due to the scatter in data from different devices, there are two concerns with this approach. Firstly the analysis relies upon the quasi-Fermi levels (and therefore carrier density) pinning above threshold and, secondly, it is assumed that the relation between internal and external currents is linear with the same slope below and above threshold, in other words that the differential injection efficiency above threshold is the same as the injection efficiency at and below threshold. The purpose of this paper is to examine these assumptions with particular reference to quantum dot lasers where there is evidence that the carrier density on the dots is not pinned above threshold.

The paper begins with a general outline of the analysis of the external differential efficiency as a function of cavity length. It is shown that, due to the onset of stimulated emission above threshold, the relation between internal and external currents is not linear going above threshold therefore it is important to distinguish "overall" efficiencies and differential efficiencies. The specific implications for quantum dot lasers are examined using calculations which replicate the non-pinning above threshold in the dots, solving the single mode photon rate equation with rate equations for occupation of dot and wetting layer states mediated by a thermal phonon distribution, without prior assumption of a Fermi-Dirac distribution. The calculated light-current curves are analyzed as functions of cavity length revealing errors in the derived values of mode loss due to this behaviour.

\section{EXTERNAL AND INTERNAL EFFICIENCY}

\section{A. The Traditional Analysis}

The equation commonly used to analyse the external differential quantum efficiency above threshold, $\eta_{e x t}^{d}$, is [1]:

$$
\frac{1}{\eta_{e x t}^{d}}=\left\{1+\frac{\alpha_{i}}{\ln \left(R^{-1}\right)} L_{c}\right\} \frac{1}{\eta_{0}^{d}}
$$

where $R$ is the power reflectivity of the facets (assumed the same at each end). A plot of $1 / \eta_{e x t}^{d}$ versus cavity length $L_{c}$ has intercept which gives the quantity $\eta_{0}^{d}$ and a slope which gives the optical mode loss if $R$ is known. $\eta_{0}^{d}$ was often interpreted as the internal efficiency or as the injection efficiency at threshold, though a number of papers has questioned these assignments, for example [2]-[4].

The basis of (1) is that, above threshold, the ratio of externally emitted intensity from both facets to the intensity in the cavity 
is given by the ratio of the total distributed mirror loss, $\alpha_{m}$, to the total optical loss, the light extraction factor [5], [24]:

$$
F=\frac{\alpha_{m}}{\alpha_{m}+\alpha_{i}}
$$

Provided the supply of carriers to the lasing states is much faster than recombination between them, the carrier density in the gain medium does not increase further above threshold because every extra electron hole pair supplied is stimulated to emit a photon by the high photon density. Consequently the carrier population and the non-radiative recombination rate do not increase and the internal differential quantum efficiency of the gain medium is one: every extra electron-hole pair produces an extra photon. This is known as Fermi level, or carrier, pinning [6], [7]. It is a consequence of the high stimulated recombination rate and occurs only where the population is inverted in the gain medium to a sufficient degree that laser action occurs.

If, above threshold, the increase in current flowing internally into the lasing gain medium is equal to the increase in the external current the relation between the internal and external differential efficiency is given simply by the light extraction factor. In these circumstances (1) follows from (2) and $\eta_{0}^{d}$ is the internal differential quantum efficiency, and the intercept of a plot of data in (1) should be one.

In practice this is not observed, $\eta_{0}^{d}$ values in the region 0.6 to 0.9 being more common and encouraging the view that this quantity is related to the efficiency at threshold [8], defined as the ratio of the internal spontaneous emission rate to the external rate of supply of electron-hole pairs (see Section V of [3] which shows the confusion at the time).

The primary reason for $\eta_{0}^{d}$ being less than one is that above threshold the current increment entering the gain medium is less than the external current increment, as was pointed out by Hakki [2] for example. For example, due to current spreading the carrier density in the gain material is not laterally uniform so there is current flow in regions where the population is not inverted and where the gain is insufficient to match the loss [9]. In these regions stimulated emission is negligible and the Fermi levels are not pinned above threshold. In quantum confined lasers the carrier density in the optical confinement layer may not be pinned above threshold and recombination in this region also reduces the current increment entering the gain medium [10].

\section{B. Differential Components Above Threshold}

Above threshold the emission is dominated by the light in the lasing mode, $L_{\text {int }}$ photons, then with reference to Fig. 1 we define an internal differential quantum efficiency of the gain medium itself as

$$
\eta_{\text {int }}^{d}=\frac{e \Delta L_{\text {int }}}{\Delta I_{\text {int }}}
$$

where $\Delta I_{\text {int }}$ is the increment in current entering the gain medium from the carrier reservoir in the barrier in response to an external current increment $\Delta I_{\text {ext }}$. The external differential

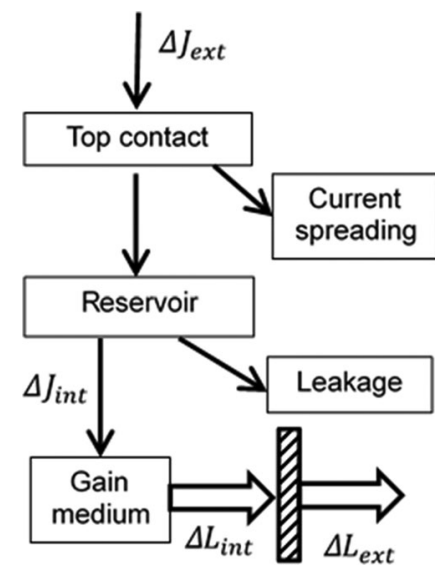

Fig. 1. Schematic diagram of current flow in a laser diode indicating the differential internal and external currents and light intensities above threshold. The reservoir may represent the wetting layer of a quantum dot laser and/or the barrier formed by the optical confinement layer. Current is lost from this region by recombination within it and leakage from it.

efficiency is

$$
\eta_{e x t}^{d}=\frac{e \Delta L_{e x t}}{\Delta I_{e x t}}
$$

The internal and external efficiencies can be related by

$$
\eta_{e x t}^{d}=\frac{e \Delta L_{e x t}}{\Delta I_{e x t}}=\frac{\Delta L_{e x t}}{\Delta L_{\mathrm{int}}} \times \frac{\Delta I_{\mathrm{int}}}{\Delta I_{e x t}} \times \frac{e \Delta L_{\mathrm{int}}}{\Delta I_{\mathrm{int}}}
$$

therefore

$$
\eta_{\text {ext }}^{d}=F \times \eta_{i n j}^{d} \times \eta_{\text {int }}^{d}
$$

where $\eta_{i n j}^{d}$ is the differential injection efficiency,

$$
\eta_{i n j}^{d}=\frac{\Delta I_{\mathrm{int}}}{\Delta I_{e x t}}
$$

and $\eta_{\text {int }}^{d}$ is as defined in (3). In general, using (2), the quantity $\eta_{0}^{d}$ in (1) is given by

$$
\eta_{0}^{d}=\eta_{\text {inj }}^{d} \times \eta_{\text {int }}^{d}
$$

If Fermi level pinning occurs $\eta_{\text {int }}^{d}=1$. Furthermore if all the extra external current enters the gain region $\eta_{i n j}^{d}=1$ and $\eta_{0}^{d}=1 \times 1=1$ : this is equivalent to saying that the Fermi levels are pinned everywhere in the device. If pinning only occurs in the lasing region $\eta_{0}^{d}=\eta_{i n j}^{d} \times 1$. This is the differential injection efficiency above threshold: to relate measured and calculated currents at threshold we require the overall injection efficiency at threshold. Is this the same as $\eta_{i n j}^{d}$ ?

\section{Efficiencies at Threshold}

At threshold the gain matches the optical loss but the photon density in the lasing mode is still small and the stimulated emission rate is assumed negligible and the radiative current in the gain material is due to spontaneous emission alone (rate $R_{\text {spon }}$ per unit area). The total internal recombination current includes non-radiative processes (rate $R_{n r}$ per unit area) in the 


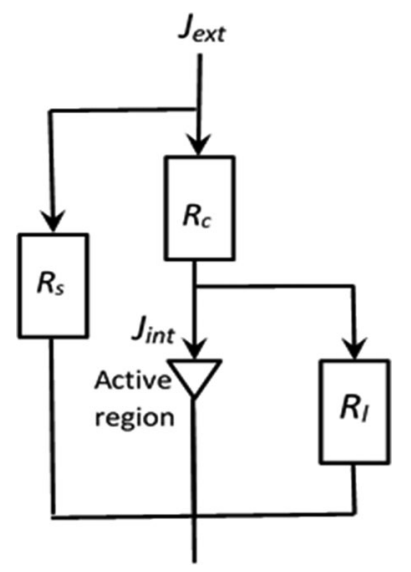

Fig. 2. A simplified equivalent circuit of a diode laser for the current spreading $R_{s}$, current leakage $R_{l}$, series contact resistance $R_{c}$, and the active region gain material.

gain medium and the internal radiative efficiency is defined as

$$
\eta_{\text {int }}^{\text {spon }}=\frac{R_{\text {spon }}}{R_{\text {spon }}+R_{n r}}=\frac{e R_{\text {spon }}}{J_{\text {int }}}
$$

This contrasts with the internal differential efficiency which is not influenced by non-radiative recombination when pinning occurs. The overall injection efficiency at threshold is

$$
\eta_{i n j}=\frac{J_{\text {int }}}{J_{\text {ext }}}
$$

and the external current at threshold is related to the internal spontaneous recombination current by

$$
J_{\text {int }}^{\text {spon }}=\left(\eta_{\text {inj }} \eta_{\text {int }}^{\text {spon }}\right) J_{\text {ext }}
$$

This shows that to relate the external current to the internal (calculated) spontaneous current it is necessary to know the internal efficiency of the gain medium and the injection efficiency. There are no simple methods to determine the internal radiative efficiency but can the injection efficiency in (10) be obtained from the differential injection efficiency obtained from $\eta_{0}^{d}$ using (1) when the Fermi levels are pinned?

\section{Injection Efficiency}

If the relation between internal and external currents is linear over all currents below and above threshold $\eta_{i n \mathrm{j}}$ would be the same at all currents and equal to the differential quantity. This can be examined using the generic equivalent circuit shown in Fig. 2 where $R_{c}, R_{s}$, and $R_{l}$ represent respectively the series resistance of the contact layers, the resistance experienced by the spreading current, and the leakage and recombination current from the reservoir.

The internal current-voltage characteristic of the gain medium is shown in Fig. 3, calculated using a single mode laser model with Fermi-Dirac statistics for the occupation of the upper and lower lasing states and including non-radiative recombination. The internal voltage is the quasi-Fermi level separation. The current increases with increasing internal voltage until threshold when stimulated emission sets in and the current increases

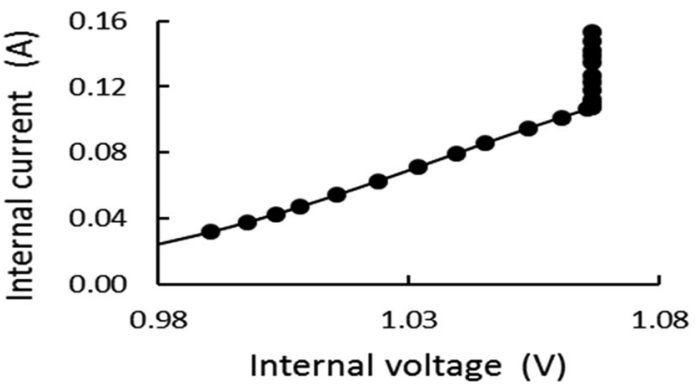

Fig. 3. Internal light current characteristic of the gain medium in a diode laser showing pinning of the voltage accompanied by a rapid increase in current due to stimulated emission above threshold. This was calculated for a quantum dot laser using a single mode laser model and Fermi-Dirac statistics for the occupation of the inhomogeneous dot states and wetting layer.

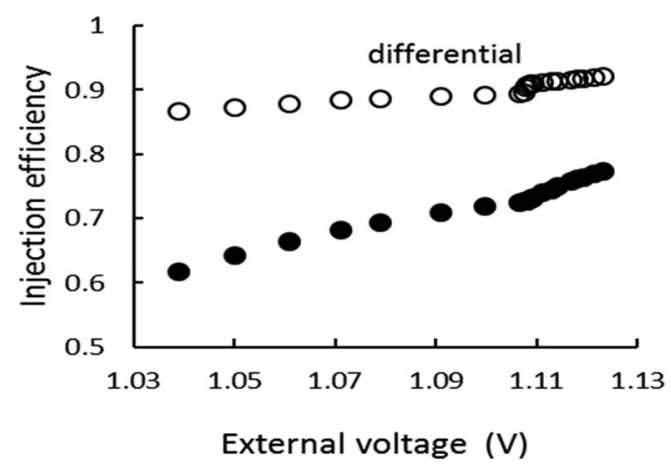

Fig. 4. Ratio of the internal and external currents, $J_{\text {int }} / J_{\text {ext }}$ (solid symbols) representing the injection efficiency, and the ratio of the current increments. $\Delta I_{\text {int }} / \Delta I_{\text {ext }}$ (open circles) representing the differential injection efficiency as functions of external voltage, calculated using the internal I-V characteristic in Fig. 3. The choice of parameter values is described in the text. The injection efficiency at threshold $(0.72)$ is not equal to the differential injection efficiency above threshold (0.92).

dramatically with negligible increase in internal voltage. This is Fermi level pinning. In this example the resistance of the gain region at threshold is about 10 ohms whereas just above threshold the differential resistance is about $10^{-3} \mathrm{ohms}$ and the gain region appears as an electrical short to increases in current above threshold.

At threshold the injection efficiency of the structure in Fig. 2 takes a value determined by the contact resistance, the resistances characterizing current spreading and leakage, and the effective resistance of the gain region. However since the gain region appears as a short to increases in current above threshold a greater fraction of the current flows through it and the differential injection efficiency above threshold is greater than the injection efficiency at threshold.

These points are illustrated in Fig. 4 for a specific numerical example. The resistive components of Fig. 2 are taken to be linear over a small voltage range in the region of threshold. Their magnitudes were chosen so that the fractions of current lost at threshold by leakage and spreading are $10 \%$ and $16 \%$ respectively, typical of broad area devices. The figure shows the injection efficiency and differential injection efficiency defined by (6) and (9) as functions of the external voltage using the 
$\mathrm{I}-\mathrm{V}$ characteristic of the gain medium in Fig. 3. The external voltage increases above threshold due to the additional voltage drop across $R_{c}$ due to the stimulated current and the differential injection efficiency rises above threshold due to the decrease in differential resistance of the gain medium. In the model of Fig. 2 the leakage current is pinned above threshold. The injection efficiency at threshold is not equal to the differential efficiency above threshold and the latter cannot be used to relate internal and external currents at threshold.

While we have used a simple equivalent circuit the key point is that the internal current-voltage characteristic of the gain material changes dramatically at threshold due to the onset of stimulated emission (this is also true for the phonon model described below) and this changes the relation between internal and external current and the assumption of a continuous linear relation at threshold and above is not valid.

\section{QUANTUM DOT LASERS}

In quantum well lasers it has been observed that although spontaneous emission from the active region of wells is pinned above threshold, the carrier density in the barrier region (or reservoir) through which the wells are is supplied is not pinned causing a reduction in the external efficiency [4]. (This is considered theoretically in [10] where the internal efficiency is taken to include the reservoir.) However in quantum dot lasers it has been reported that the spontaneous emission from the active region of dots is not pinned above threshold but continues to increase with current, albeit at a slower rate [11], [12], despite the fast stimulated emission process. This absence of pinning in the gain medium itself raises questions regarding interpretation of the external differential efficiency and the use of (1) to determine the internal mode loss.

These matters are examined using a model which replicates the increase in spontaneous emission above threshold by avoiding the prior assumption of the applicability of Fermi-Dirac statistics to these structures. The external differential efficiency is obtained from calculated light-current plots and anaysed using (1) to obtain a value for $\alpha_{i}$ that can be compared with the input value. If $\alpha_{i}$ is dependent upon the density of free carriers in the active region this also introduces a dependence of $\alpha_{i}$ on cavity length which itself invalidates use of (1) [13]. In this work we keep $\alpha_{i}$ constant and focus on the effect of the absence of pinning of emission from the dots themselves on the external differential efficiency.

\section{A. The Model}

The threshold current of quantum dot lasers between $10 \mathrm{~K}$ and room temperature has a minimum in the region of 150 $200 \mathrm{~K}$ [14] and at low temperature dots states are populated randomly with the same probability irrespective of their energy [15]. The temperature dependence of threshold is well described by a model in which the capture and emission of electrons on dot states from and to the wetting letting layer is controlled by emission and absorption of phonons in a Bose-Einstein distribution [16]. Such a model shows the transition from random population at low temperature to a Fermi-Dirac distribution at room temperature [17] and a thermal distribution is widely assumed in the calculation of gain-current relations of quantum dot lasers.

Calculations of threshold current from gain-current relations do not include stimulated emission, since it is negligible just at threshold, so to explore the behavior above threshold it is necessary to take account of stimulated emission. This has been done by combining the electron-phonon model for state occupation (as in [17]) with a single mode laser model for the interactions between electrons and photons. The ground and excited states of InAs dots are populated by electron hole pairs at a rate determined by the capture and emission of electrons from and to the wetting layer by interaction with a Bose-Einstein phonon distribution (see [18]) with the same rate constants as used to describe the temperature dependence of threshold in [17]. The dot density was $3 \times 10^{10} \mathrm{~cm}^{-2}$ in an inhomogeneous distribution with standard deviation of $20 \mathrm{meV}$ and the optical cross section of ground and excited states was $1.2 \times 10^{-13} \mathrm{~cm}^{2}$ from absorption measurements [17].

Non-radiative recombination in the dots and radiative $\left(\propto n_{w l}^{2}\right)$ and non-radiative $\left(\propto n_{w l}\right)$ processes in the wetting layer were included. Since the wetting layer states are very closely spaced their carrier distribution was described by FermiDirac statistics. The wetting layer is populated via the waveguide and carriers may be lost by recombination in the core and leakage through the cladding layer. The rate-limiting step in the supply of carriers to the dots is assumed to be the coupling between wetting layer and dots, therefore for the purposes of this paper these waveguide processes are combined in a thermally induced carrier leakage controlled by a barrier relative to the wetting layer quasi-Fermi level with a linear prefactor chosen to give a contribution of about $7 \%$ of the total current at threshold.

Current spreading was described by the model of Tsang [19, eq. (4)] with parameters chosen to give a spreading current in the region of $10 \%$ of the total current at threshold. For comparison, calculations were also done with a Fermi-Dirac distribution across ground and excited states and wetting layer. The facet power reflectivity and optical mode loss were 0.3 and $3 \mathrm{~cm}^{-1}$ respectively for all cavity lengths. The calculations were done at $300 \mathrm{~K}$.

The phonon-induced emission from dot states depends on their energy relative to the wetting layer therefore their occupancy varies across the inhomogeneous distribution. The rate equations were applied to 21 groups of different size dots interacting with the same carrier density in the wetting layer. The spontaneous emission, stimulated emission and recombination current were summed over all dots to give the internal characteristics of the dot system then leakage and spreading currents obtained as described above and the light extraction factor applied to give the external characteristics.

\section{B. Calculated Light-Current Curves}

The light current curve for a $3 \mathrm{~mm}$ long laser in Fig. 5 shows the same threshold for the phonon-mediated and Fermi Dirac calculations, but the external differential efficiency for the phonon-mediated case is smaller (0.48) than the FermiDirac calculation (0.54). Higher order contributions to the waveguide carrier loss would reduce the value of $\eta_{e x t}^{d}$ for the 


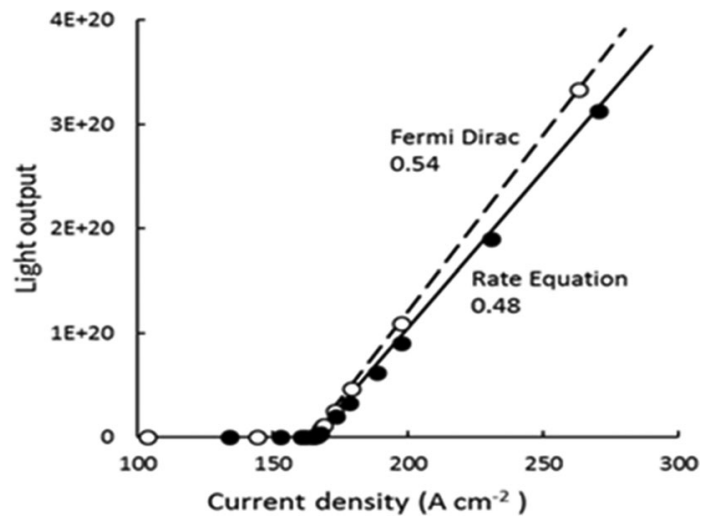

Fig. 5. Light-current plots calculated for a $3 \mathrm{~mm}$ long device using phononmeditated rate equations and Fermi-Dirac statistics. Both give the same threshold current but the phonon model has a lower external differential efficiency, given by the numbers adjacent to the plots.

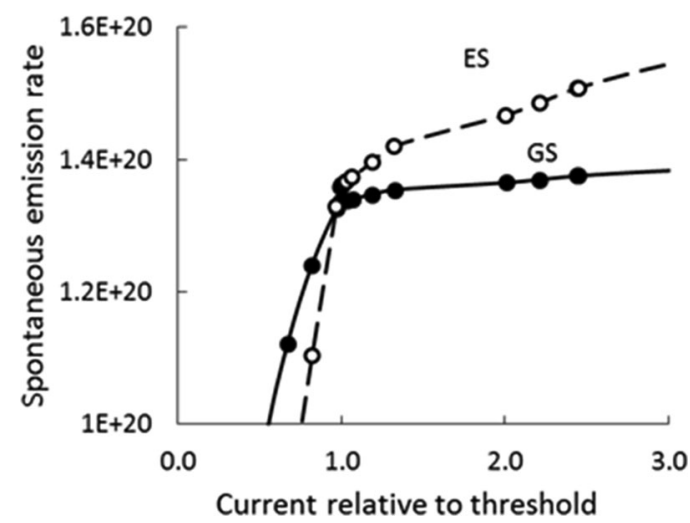

Fig. 6. Spontaneous emission from ground and excited states, summed over all dots, as functions of current relative to threshold calculated using the phonon model, showing the emission is not pinned above threshold. In this example the excited state emission exceeds that from the ground state because the former has a degeneracy twice that of the ground state.

phonon-mediated calculation. Values in the region of 0.5 are reported for the external differential efficiency in [11]. Fig. 6, for the phonon model, shows the spontaneous emission from the ground and excited states increases above threshold similar to the observations [11] whereas using Fermi statistics the emission is pinned.

\section{Occupation Probability Distributions}

The occupation probabilities of overlapping inhomogeneous distributions of ground and excited states are shown in Fig. 7 as functions of dot state energy below the wetting layer.

The open symbols are for the phonon calculation at threshold and show ground and excited states to be in equilibrium with each other. The lower solid line is the Fermi-Dirac distribution if the dot states were in quasi-equilibrium with the wetting layer: this agrees with the phonon calculation.

The solid symbols are the occupation probabilities from the phonon calculation above threshold (about $5 \times I_{\text {th }}$ ). The occupation of all dot states has increased except those

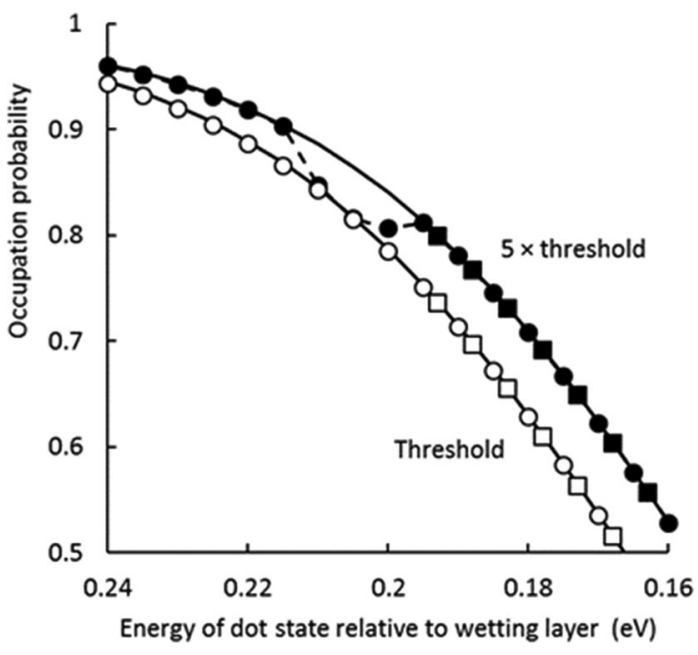

Fig. 7. The symbols show the ground (circles) and excited (squares) state occupations as functions of the state energy below the wetting layer calculated on the phonon model. The lines are the dot state occupation given by a FermiDirac distribution with the Fermi energy of the wetting layer. At threshold (open symbols) the ground and excited states and the wetting layer are in quasithermal equilibrium. Above threshold (solid symbols) the occupation of states in the region of $0.21 \mathrm{eV}$ below the wetting layer is pinned at the threshold value; that of all other states has increased, as has the carrier density of the wetting layer, evidenced here by the higher Fermi energy identified as the energy where the probability is 0.5 .

responsible for laser action near the peak of the inhomogeneous distribution (about $0.21 \mathrm{eV}$ below the wetting layer). For these states the occupation is pinned at its threshold value. The higher occupation of the non-lasing states is responsible for the increasing spontaneous emission above threshold. The wetting layer carrier density increases above threshold, as does the carrier loss via the optical confinement layer, and the upper solid line is the Fermi distribution which would arise if the dot states were in quasi-equilibrium with this increased population: the occupation of all dot states, other than those which are lasing, is in quasi-equilibrium with the wetting layer population.

The Figure shows that above threshold (i) occupation of those states responsible for lasing is pinned, (ii) occupation of nonlasing states is in quasi-equilibrium with the wetting layer, and (iii) the populations of the wetting layer and non-lasing states are not pinned and increase with current. Similar behaviour is apparent in the calculation of Chow et al. [20].

\section{The Role of Stimulated Emission}

Carriers are supplied to the dot states by capture from the wetting layer and the capture time constant, $\tau_{c a p}$, into an unoccupied state is not strongly dependent on the energy of the state. Carriers are lost from the dot state by non-radiative, spontaneous and stimulated recombination with holes in the same dot and by emission to the wetting layer by absorption of a phonon. The time constant for emission to the wetting layer $\tau_{e m}$ decreases with decreasing energy separation between states and wetting layer. We represent spontaneous and non-radiative recombination by their combined rate $\tau_{r e c}^{-1}$. 
In a dot which is not contributing to lasing the stimulated recombination rate is negligible compared with $1 / \tau_{\text {rec }}$ and $1 / \tau_{\text {rec }}$ $<<1 / \tau_{\text {em }}$. The occupation in a non-lasing dot is therefore determined by competition between phonon-mediated capture and emission [18]:

$$
f_{n l} \cong \frac{1}{1+\frac{\tau_{c a p}}{\tau_{e m}}}
$$

Since the interaction with the wetting layer occurs at rates greater than the recombination rate $f_{n l}$ is a Fermi distribution.

In a lasing dot the stimulated recombination rate $\left(1 / \tau_{\text {stim }}\right)$ is very high and $1 / \tau_{\text {rec }}, 1 / \tau_{\text {em }} \ll 1 / \tau_{\text {stim }}$ so the average occupation of a lasing state is determined by competition between capture and stimulated recombination:

$$
f_{l} \cong \frac{1}{1+\frac{\tau_{c a p}}{\tau_{s t i m}}}
$$

As the photon density increases above threshold the stimulated rate increases. This rate is limited by the rate at which carriers are captured onto the dot state which in turn is proportional to the carrier density in the wetting layer, $n_{w l}$. Therefore as the external current is increased above threshold the wetting layer carrier density increases, increasing the rate of supply of carriers to the dot, enabling the stimulated rate and light output to increase. As the current is increased $n_{w l}$ increases to maintain the dot occupation necessary to produce the required optical gain. The occupation of the lasing states is therefore pinned above threshold by the stimulated rate.

The wetting layer carrier distribution is internally in quasiequilibrium, therefore the increase in $n_{w l}$ above threshold also increases the capture rate to the non-lasing dots. Since their emission rate to the wetting layer remains the same (11) shows that their occupation increases and remains in quasi-equilibrium with the wetting layer resulting in increasing spontaneous emission from non-lasing dots.

\section{EFFICIENCY OF QUANTUM DOT LASERS}

\section{A. Analysis of Length Dependence}

The external differential efficiencies for a range of cavity lengths were obtained from light-current curves calculated using the phonon-mediated model and their reciprocals are plotted versus cavity length in Fig. 8. Analysis according to (1) gives $\eta_{0}^{d}=0.80$ from the intercept and $\propto_{i}=2.57 \mathrm{~cm}^{-1}$ from the slope. The latter deviates from the value of $3 \mathrm{~cm}^{-1}$ input to the calculations and shows that errors can arise in determination of mode loss by this method when the carrier densities are not pinned throughout the device, even though $\propto_{i}$ remains constant.

Table I gives calculated values of differential internal and injection efficiencies just above threshold for the whole dot system. This reveals two key points: the internal differential efficiency of the dot system is less than 1 and the efficiencies vary with cavity length. The latter shows that $\eta_{\text {int }}^{d}$, while being "internal" to the dot system, is nevertheless influenced by the length of the device because the number of lasing dots and carrier densities of non-lasing dots vary with the gain requirement. Furthermore the quantity $\eta_{0}^{d}$ obtained from Fig. 8 over a

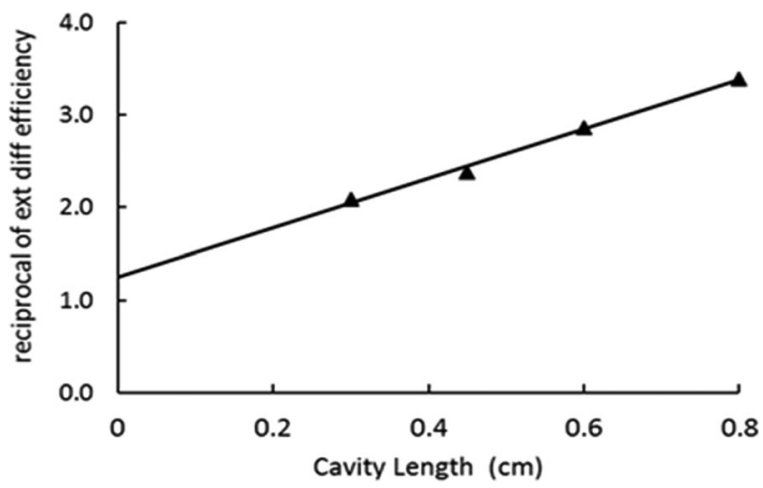

Fig. 8. Plot of the reciprocal of the external differential efficiency from lightcurrent plots calculated using the phonon rate equation model as a function of cavity length.

TABLE I

CAlculated DifFerential EFFicIENCIES For Dot System

\begin{tabular}{lccc}
\hline \hline Cavity length, mm & $\eta_{i n j}^{d}$ & $\eta_{\text {int }}^{d}$ & F \\
\hline 300 & 0.87 & 0.98 & 0.571 \\
800 & 0.91 & 0.97 & 0.333 \\
\hline
\end{tabular}

range of cavity lengths cannot formally be interpreted in terms of efficiencies of a specific structure. To understand these two points requires consideration of what should be regarded as the active lasing region in these devices, and this has implications for interpretation of data for external efficiency and using it to relate experiment and theory.

\section{B. Definitions}

Not all dot states have their occupation pinned above threshold. The occupation of those dots contributing to laser action is pinned by the gain required and their internal differential efficiency above threshold is one. The external differential efficiency (5) is then the product of the light extraction factor and the differential injection efficiency into the subset of dots which are lasing. This subset constitutes the active region. However, as the current and wetting layer carrier density increase above threshold the occupation of adjacent size groups in the dot distribution becomes sufficient for them generate sufficient gain and produce stimulated emission. The occupation of these dots is then pinned and the number of dots in the lasing subset increases with increasing current. This results in broadening the lasing spectrum [21], [22]. While this increase in the population of lasing dots is inherent in a calculation such as described above, there are implications for interpretation of experimental differential efficiency data.

The dots interact with the wetting layer and one approach is to take the active region as the whole inhomogeneous ensemble of dots and wetting layer ( $\mathrm{D}+\mathrm{WL})$, as is in some gain calculations, eg [23]. Under this definition the differential injection efficiency is the fraction of the external current increment which enters the D+WL system and the "internal" current increment above threshold into $\mathrm{D}+\mathrm{WL}$ includes an increase in recombination 
TABLE II

DIFFERENTIAL EFFICIENCIES, $L_{\mathrm{c}}=300 \mathrm{~mm}$

\begin{tabular}{lcc}
\hline \hline Active region & $\eta_{i n j}^{d}$ & $\eta_{\text {int }}^{d}$ \\
\hline Dots & 0.87 & 0.98 \\
Dots + Wetting layer & 0.94 & 0.90 \\
\hline
\end{tabular}

in the non-lasing dots and in the wetting layer. The internal differential efficiency of this $\mathrm{D}+\mathrm{WL}$ region is less than one because some carriers increase the carrier density in the nonlasing dots and wetting layer rather than producing photons.

Alternatively the active region can be taken to be the inhomogeneous dot system alone (as in Table I). The differential injection efficiency is then the fraction of the external current increment that enters the dot system only and its internal differential efficiency is slightly less than one due to the increasing occupation of non-lasing dots above threshold, as shown in Table I.

Neither of these definitions is ideal because the carrier density is not pinned throughout the specified active region so in each case $\eta_{\text {int }}^{d}<1$. However the measured external differential efficiency is a property of the device and is not affected by the region we choose to designate as "active" in our interpretation of the results. It is important therefore to adopt consistent definitions of differential injection and internal efficiency when interpreting experimental data to compare experiment and theory.

\section{Choice of Active Region}

Table II gives values for differential injection and internal efficiency for a $3 \mathrm{~mm}$ long device when the active region is chosen to be the dots alone, and the dot system plus wetting layer. The injection efficiency of the dots alone is less than that of combined dots and wetting layer whereas the internal efficiency of the combined D+WL system is lower than the dots alone and this is due to the current flow by recombination in wetting layer increasing the injection efficiency but decreasing the internal efficiency of the combined system. When combined with the light extraction factor both systems give the same external differential efficiency of 0.48 . These data indicate that the dots-alone definition has a differential internal efficiency close to one (0.98), so the interpretation of $\eta_{0}^{d}$ as the differential injection efficiency $(=0.8)$ may be a reasonable approximation; the same cannot be said of the dots plus wetting layer definition.

\section{Discussion}

The competition between phonon mediated transfer of carriers between dots and wetting layer and stimulated emission has two effects: the spontaneous emission from the dot system is not pinned reducing its differential internal efficiency, and the carrier density in the wetting layer and associated recombination and leakage currents are not pinned, reducing the differential injection efficiency. Since the stimulated rate per dot is many orders greater than the spontaneous rate per dot the absence of pinning in the dot system has a small effect on the internal efficiency of the dots, reducing $\eta_{\text {int }}^{d}$ by only a few percent (Table I).

The effect of non-pinning in the wetting layer on $\eta_{i n j}^{d}$ is somewhat greater and leads to further un-pinned currents due to waveguide carrier loss and spreading. In designing devices it is desirable to minimize these to reduce the threshold current and maximize the external differential efficiency. There are wellknown strategies to do this such as minimizing the density of defect-related recombination centres and maximizing the barrier to carrier leakage, however the fundamental origin of this behavior is the increase in wetting layer carrier density necessary to maintain a capture rate to match the stimulated emission rate at each dot. For a given optical loss the stimulated rate per dot can be reduced by increasing the number of dots, for example by growing more dot layers, reducing the net capture rate per dot necessary for lasing, and hence the wetting layer carrier density.

This has been confirmed by repeating the calculations for the $3 \mathrm{~mm}$ long laser with twice the dot density; this increases the external differential efficiency from 0.48 to 0.53 for the parameter values used here. The differential injection efficiency is increased from 0.87 to 0.94 and the overall efficiency at threshold is also increased reducing the total threshold current density.

\section{SUMMARY AND CONCLUSIONS}

We have reviewed the traditional analysis of the length dependence of the external differential efficiency. If the carrier density is pinned in the active region such that the internal differential efficiency above threshold is one then the analysis gives the differential injection efficiency. However, due to the effect of stimulated emission on the internal current-voltage relation of the active region this differential injection efficiency above threshold is not equal to the overall injection efficiency at threshold and cannot be used to relate the internal radiative current to the external current of a device.

Using a model for phonon-mediated exchange of carriers between dots and wetting layer coupled to a single mode laser rate equation, we have reproduced the observed increase in spontaneous emission from dots above threshold, which does not occur using Fermi-Dirac statistics. The threshold currents calculated by the two models agree and at threshold the state occupation probability distribution of dots and wetting layer calculated on the phonon model corresponds to a Fermi-Dirac distribution. However the phonon model shows that above threshold the high stimulated emission rate in the lasing dots pins their occupation whereas occupation of the non-lasing dots and their spontaneous emission increases with current.

When comparing experiment and theory it is important to identify the internal "active region" for which the laser properties have been calculated and to use this to make a consistent interpretation of experimental data. Whether the active region is regarded as the dot system, or the dot system and wetting layer, its internal differential quantum efficiency is less than one because laser action does not occur at all states within it. Furthermore the differential injection and internal efficiency 
depend on cavity length and the internal mode loss obtained from analysis of the calculated external differential efficiency does not agree with the input value.

The key implications of this work are that Fermi-Dirac based calculations do not provide a good representation of quantum dot lasers above threshold and are likely to overestimate the external slope efficiency and light output of high power dot lasers. The value of optical mode loss obtained from the length dependence of the measured external efficiency will be in error due to this as well as other causes.

\section{ACKNOWLEDGMENT}

The author would like to thank P. Smowton, I. O'Driscoll, and $\mathrm{S}$. Shutts for valuable discussions and comments.

\section{REFERENCES}

[1] J. R. Baird, W. N. Carr, and B. S. Read, "Analysis of a GaAs laser," Trans. Amer. Inst. Mining, Metall. Petroleum Eng., vol. 230, pp. 286-290, Mar. 1964.

[2] B. W. Hakki, "GaAs double heterostructure lasing behavior along the junction plane," J. Appl. Phys., vol. 46, pp. 292-302, 1975.

[3] F. R. Nash and R. L. Hartman, "Facet oxide formation and degradation of GaAs lasers," J. Appl. Phys., vol. 50, no. 5, pp. 3133-3141, May 1978.

[4] P. M. Smowton and P. Blood, "The differential efficiency of quantum well lasers," IEEE J. Sel. Topics Quantum Electron., vol. 3, no. 2, pp. 491-498, Apr. 1997.

[5] H. C. Casey and M. Panish, Heterostructure Lasers. San Diego, CA, USA: Academic, 1978, eqs. 3.8-31.

[6] L. A. Coldren, S. W. Corzine, and M. L. Masanovic, Diode Lasers and Photonic Integrated Circuits. New York, NY, USA: Wiley, 2012, sec. 5.2.2.3.

[7] P. Blood, Quantum Confined Laser Devices. London, U.K.: Oxford Univ. Press, 2015, sec. 5.2.4.

[8] G. H. B. Thompspon, G. D. Henshall, J. E. A. Whiteaway, and P. A Kirkby, "Narrow-beam, five-layer GaAlAs/GaAs heterostructure lasers with low threshold and high peak power," J. Appl. Phys. vol. 47, pp. 1501-1514, May 1976.

[9] P. Blood, Quantum Confined Laser Devices. London, U.K.: Oxford Univ. Press, 2015, sec. 14.2.

[10] L. V. Asryan, S. Luri, and R. A. Suris, "Internal quantum efficiency of semiconductor lasers with a quantum confined active region," IEEE J. Quantum Electron., vol. 39, no. 3, pp. 404-418, Mar. 2003.

[11] I. P. Marko, A. R. Adams, N. F. Masse, and S. Sweeney, "Effect of nonpinned carrier density above threshold in InAs quantum dot and quantum dash lasers," IET Optoelectron., vol. 8, no. 2, pp. 88-93, Apr. 2014.

[12] P. M. Smowton, private communication.
[13] L. V. Asryan, "Limitations on standard procedure of determining the internal loss and efficiency in quantum dot lasers," J. Appl. Phys., vol. 99, 2006, Art. no. 013102.

[14] A. E. Zhukov et al., "Negative characteristic temperature of InGaAs quantum dot injection laser," Jap. J. Appl. Phys., vol. 36, pp. 4216-4218, 1997.

[15] M. Grundmann and D. Bimberg, "Theory for random population for quantum dots," Phys. Rev. B, vol. 55, pp. 9740-9745, Apr. 1997.

[16] H. Huang and D. G. Deppe, "Rate equation model for non-equilibrium operating conditions in a self organized quantum dot laser,' IEEE J. Quantum Electron., vol. 37, no. 5, pp. 691-698, May 2001.

[17] I. O’Driscoll, M. Hutchings, P. M. Smowton, and P. Blood, "Random population of quantum dots in InAs-GaAs laser structures," IEEE J. Quantum Electron., vol. 37, no. 4, pp. 525-532, Apr. 2010.

[18] P. Blood, Quantum Confined Laser Devices. London, U.K.: Oxford Univ. Press, 2015, ch. 10.

[19] W. T. Tsang, "The effects of lateral current spreading, current outdiffusion, and optical mode losses on the threshold current density of GaAs-AlGaAs stripe geometry lasers," J. Appl. Phys., vol. 49, pp. 10311044, Mar. 1978.

[20] W. W. Chow, F. Jahnke, and C. Gies, "Emission properties of nanolasers during the transition to lasing," Light Sci. Appl., vol. 3, Aug. 2014, Art. no. e201.

[21] M. Sugawara, K. Mukai, Y. Nakata, K. Otsubo, and H. Ishikawa, "Performance and analysis of quantum dot lasers with self-assembled columnarshaped and $1.3 \mu \mathrm{m}$ emitting quantum dots," IEEE J. Sel. Topics Quantum Electron., vol. 6, no. 3, pp. 462-474, May/Jun. 2000.

[22] P. Finch et al., "Improving the optical bandwidth of passively mode-locked InAs quantum dot lasers," IEEE J. Sel. Topics Quantum Electron., vol. 21, no. 6, Nov./Dec. 2015, Art. no. 1900507.

[23] H. C. Schneider, W. W. Chow, and S.W. Koch, "Many-body effects in the gain spectra of highly excited quantum dot lasers," Phys. Rev. B, vol. 64, Aug. 2001, Art. no. 115315.

[24] P. Blood, Quantum Confined Laser Devices. London, U.K.: Oxford Univ. Press, 2015, sec. 5.5.2.

Peter Blood worked previously at Philips Research Laboratories, Redhill, U.K., and as a visitor at Bell Laboratories, Murray Hill, USA. His research activities at this time included ion implantation and studies of deep states in Si and in III-V compounds. Since 1983, he has been conducting research into quantum well structures, and, in 1990, he moved to Cardiff working on the physics quantum well lasers, later on quantum dot devices where his current research activity is focused. The group has developed spontaneous emission and stripe length techniques for the study of recombination and optical gain, which provide a full characterization of gain media. He has coauthored two books on the characterization of semiconductors and has recently published a single author book on Quantum Confined Laser Devices.

Dr. Blood served as an Associate Editor for the Journal of Quantum Electronics and has given tutorials and short courses at numerous international conferences. He is a Fellow of the Institute of Physics. He is an Honorary Distinguished Professor in the School of Physics and Astronomy in Cardiff University. 